\title{
An Improved OVSF Code Assignment Scheme at the Forward Link of WCDMA 3G System
}

\author{
Mala Umar Mustapha Bakura, Jing XiaoJun, and Sun Songlin
}

\begin{abstract}
CDMA is the access technique adopted in the third generation wireless system. In this technique, all users share the same bandwidth simultaneously but with different codes. This sharing generates interferences that can reduce the overall throughput of the WCDMA network when using a weak code allocation algorithm. In this paper we propose a code management and allocation scheme that ensures to a great extend an unrestricted access to the Wideband Code Division Multiple Access (WCDMA) network and greatly reducing the fragmentation of the code tree. Rather than simply keeping the code tree as compact as possible by merely depending on the request rate, we design an improved Region Division Allocation (IRDA) of OVSF codes a scheme for OVSF code tree management for the universal mobile telecommunication system (UMTS) by considering characteristics of traffic and dynamic resource allocation (DRA) to make the management efficient and satisfy different quality-of-service (QoS) requirements requested by diverse multimedia. According to our proposal, users would not only be restricted to their pre-defined code region but are allowed to borrow from other code regions and return to their home code if released by initial users. The simulation result indicates that optimum and fair usage of the available code was achieved
\end{abstract}

Index Terms-Code blocking, fragmentation, OVSF Code, spreading factor, WCDMA.

\section{INTRODUCTION}

The second generation (2G) mobile communication systems provide voice and low-rate data transmission, while the third generation (3G) network is targeted to provide high-rate transmission for multimedia applications. To fulfill the goal, UMTS employs wide-band code division multiple access (WCDMA) to support multi-rate applications utilizing OVSF codes. This technology gives user elements UE the ability to have full duplex access either through frequency division duplex FDD or time division duplex DD. In the FDD mode, separate $5 \mathrm{MHz}$ carrier frequencies are used for the uplink and downlink respectively, whereas in TDD only one $5 \mathrm{MHz}$ band is time shared between the uplink and downlink. The third generation networks answer the needs for increasing

Manuscript received March 20, 2012, revised April 3, 2012. This work was supported by School of Information and Communications Engineering, Beijing University of Posts and Telecommunications and Key Laboratory of Trustworthy Distributed Computing and Service (BUPT), Ministry of Education, Beijing University of Posts and Telecommunications.

Authors are with Beijing Univeristy of Post and Telecommunication. No: 10 Xitu Cheng Lu, Haidian District. Beijing-100876, China (e-mail: bkumar52@hotmail.com; jxiaojun@bupt.edu.cn; slsun@bupt.edu.cn). demand for high and variable data rate services such as video, web surfing, image and data communication. In the downlink of WCDMA, variable data rate is supported by using orthogonal variable spreading factor OVSF codes. The codes have different spreading factors or chip rates in that higher user data rates are spread using low spreading factor SF and lower user data rates are spread using high SF. In either case, the user data rate is spread to attain the bandwidth of $5 \mathrm{MHz}$. Apart from spreading, the OVSF codes also maintains orthoganality between different users in the sense that the correlation between the sub channels is zero which indicates that there is no interference between the sub channels. Mathematically it is represented as.

$$
\{f(x) \lg (x)\} \equiv \int_{a}^{b} f(x) g(x) d x=0, a \leq x \leq b
$$

The equation gives the convolution or cross-correlation between the two functions $f(x)$ and $g(x)$ so as to determine the relation between them which is also known as sliding dot product.

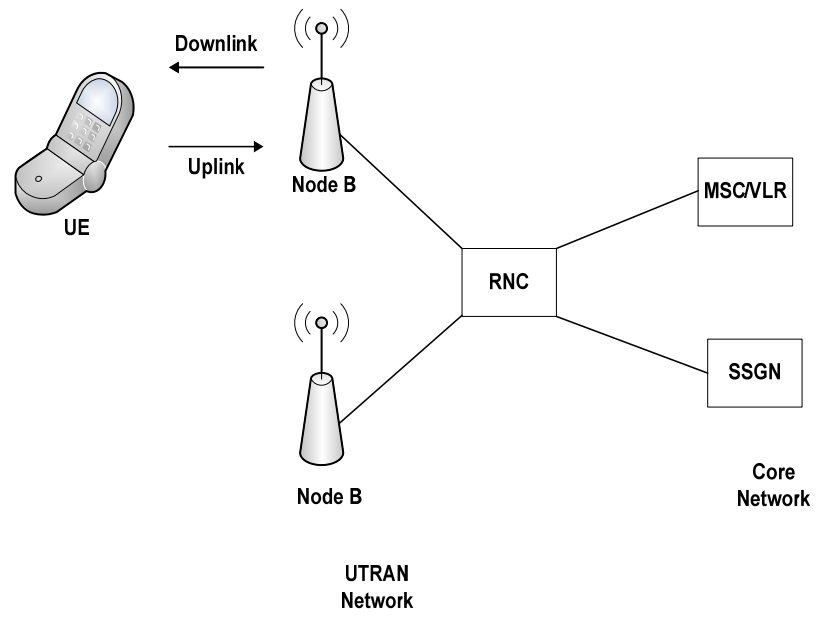

Fig. 1. UMTS network

This result explains the fact that the interference between two or more channels of the same cell is zero. Another important issue in code assignment is code blocking a phenomenon where an incoming call is blocked not due to lack of transmission channel but due to lack of OVSF code caused mostly by fragmentation in the code tree. Code assignment schemes should fairly [1] assign codes to Low data rate sessions and High data rates session while 
considering existing interference before assignment. The rest of the paper is organized as follows. Section 2 discusses the OVSF code tree, section 3 summarizes previous work, section 4 for other code allocation schemes, Section 5, deals with IRDA, section 6 deals with simulation and result while section 7 is for conclusion.

\section{OVSF CODE TREE}

All Hadamard-Walsh matrix is used to generate a successive orthogonal variable codes which can be represented by the tree shown in Fig. 2

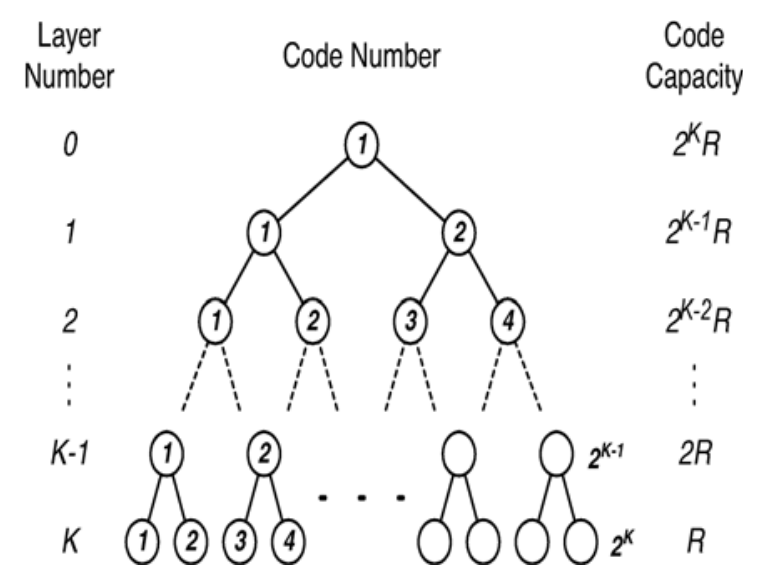

Fig. 2. OVSF code tree

Code length changes from layer to layer. The tree has a $\mathrm{k}^{\text {th }}$ -layered structure. The topmost layer $\left(0^{\text {th }}\right.$ layer $)$ having the least spreading factor (SF) is the root of the tree while the lowest layer $\left(\mathrm{k}^{\text {th }}\right.$ layer $)$ with highest $\mathrm{SF}$ is the leave of the tree. The number of codes in the leave which corresponds to its SF represents the capacity of the whole tree. In other word the SF of the leave indicates the number of calls the tree can support.

All lower layer codes linking a particular code to the root code are called the mother codes while all higher layer codes that originate from the lower layer code are named child codes. Each code in the tree can be represented as $C_{(S F, N)} 1 \leq n \leq S F$ where SF is the spreading factor of the code and $\mathrm{n}$ indicates the position of the code in the layer. The spreading factor is the number of chips used to represent each data bit. Numbers encircled in Fig 2 indicates the position of the code in that particular layer which is $\mathbf{n}$. All codes in the same layer are mutually orthogonal, also all codes in different layer are orthogonal too if they do not have mother child relationship. The maximum capacity of the system is expressed as Capacity $=2^{K} R . K$ is the highest layer of the tree and $\mathrm{R}$ is the fundamental data rate. It is obvious from the tree that as we move up from the leaves towards the root $0^{\text {th }}$ layer, higher bit rate are supported but the tree shrinks exponentially in terms of available codes.

\section{A. Code Segmentation and Blocking}

After a period of operation, available codes will be scattered around the code tree. The random scattering of the available codes within the code tree known as fragmentation results in code blocking. This affects the performance of the system. Code blocking is phenomena where by a call or session is blocked even though the system has enough capacity to support the rate requirement of the call or session. As can be observed in Fig. 3, below, the subtree can support a call that does not exceed $4 \mathrm{R}$ bps data rate. This is determined by checking whether or not the Kraft's inequality $\sum_{i=1}^{l} 2^{-r} \leq 1$ is satisfied or by simply counting the number available orthogonal codes marked with diagonal stripes in the $\mathrm{R}$ bps layer i.e in the SF 8 layer. In this case we have a total of 4R bps. Despite this, if a call requiring a single code of SF 2 is blockenbecause the two SF 2 codes marked with vertical stripe in Fig. 3 are all blocked. A situation known as code blocking occurs. On the other hand four calls each of R are fully accommodated by the tree because the four codes represented by the diagonal stripes are assignable. This also explains what we mean by skewing of the code tree. Where a higher data rate is blocked in preference to lower bit rate calls.

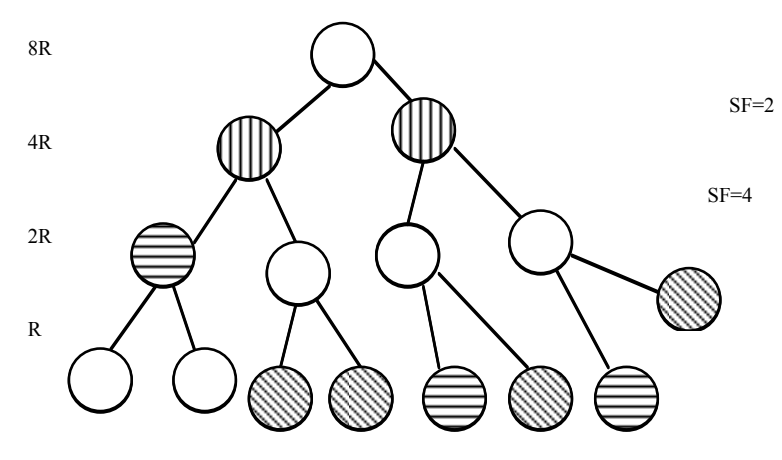

Fig. 3. Code blocking

\section{RELATED WORK}

In the downlink of WCDMA several channels of variable data rates are transmitted at the same time. The variable data rates are supported by using a single orthogonal variable spreading factor code to spread and maintain an interference free communication. A great deal of research is done to ensure an efficient use of the available codes in order to accommodate the highest number of channels possible at the same time, also to reduce fragmentation within the code tree. The code assignment can largely be grouped into conventional allocation, dynamic allocation, region division/partitioning (dynamic partitioning) allocation, Hybrid code allocation, genetic Algorithm allocation and others.

\section{B. Conventional Code Allocation Scheme}

In this code assignment scheme the code allocation is simple and straight forward. It is a system in which in-coming calls or sessions are allowed to transmit if their requested codes are available and assignable if no assignable code available, the call is instantly blocked. This assignment scheme is very simple to implement but has greater probability of code block. 


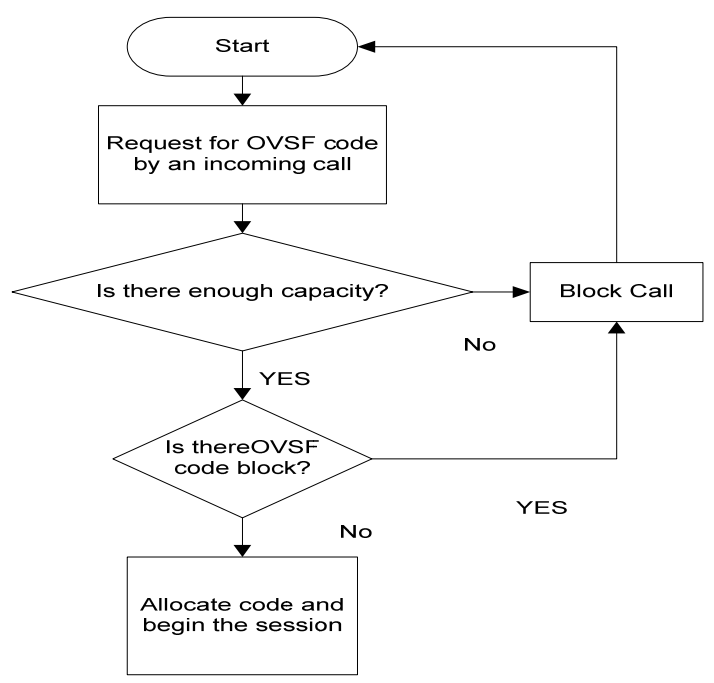

Fig. 4. CCA flow chart

\section{C.Dynamic Code Allocation Scheme (DCA)}

As the name suggest, the allocated codes are dynamic in nature in the sense that a particular session may start transmitting with a particular OVSF code and end its session with a different code [2]. As earlier stated, if the available assignable codes satisfy the Kraft's inequality it implies that the call can be supported by the channelization code. In the event where there is no single code available to support this particular call session as explained in example 2.1 above, a reassignment of codes takes place to create room to accommodate this call. The spreading code of one or more calls are interchanged with new codes so that an assignable code that meet the spreading and orthogonality requirements of the new call is created thereby. The DCA [2] ensures efficient use of the channel resources with more throughput and little blocking but comes with it an additional signaling and delay.

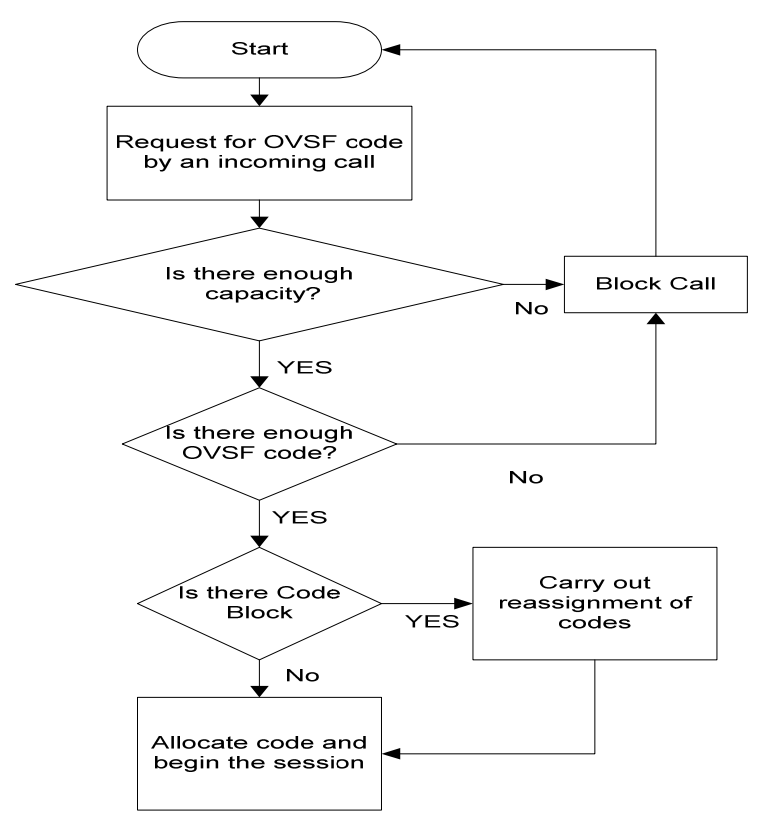

Fig. 5. DCA flow chart

\section{Optimum DCA}

Since the amount of time needed to do the reassignment of code has limit, optimum DCA is a proposal that considers each reassignment as a cost such that the network is made to have a threshold value for this cost. Therefore for a new call to be admitted three conditions need to be fulfilled. These are the available resources or the channel capacity, satisfaction of Kraft's inequality and number of code reassignment needed. Therefore Optimum DCA gives a relatively good performance compared to DCA under a more realistic scenario.

\section{E. Code Partitioning Scheme}

Under this scheme the whole OVSF code tree is partitioned into regions just like RDA but the partitioning is further classified as either complete source CS, complete partition $\mathrm{CP}$ or hybrid partition HP. In the complete partitioning, a distinct partition is reserved for a distinct user data rate while the hybrid partition HP is of two types. One is HP which involves the combination of CS and CP and the second is $\mathrm{CP}$ and rate sharing. Rated sharing involves allocating specific rated neighbor partitions from which a particular partition can borrow codes from. Details are contained in [3]. CS is simply the same with conventional code allocation where the whole code tree serves as a pool from where code is selected from.

RDA and Code partitioning scheme address the problem of skewing in code selection. Skewing is a phenomenon where low data rate calls are preferentially allocated codes compared to high data rate calls

\section{F. Hybrid Code Allocation (HCA)}

In this allocation method the advantages offered by both RDA and DCA are utilized to yield a better performance in increasing throughput, fair allocation and reducing code blocking. HCA [4] firstly carry out RDA and in the case of blocking a local DCA is carried out as explained in the flow chart of Fig. 6. Another Code Channel Assignment with Dynamic Partitioning and Buffering CADPB was proposed. This is a method where the partition is not fixed. The partition varies based on the statistics of call at that particular time [5].

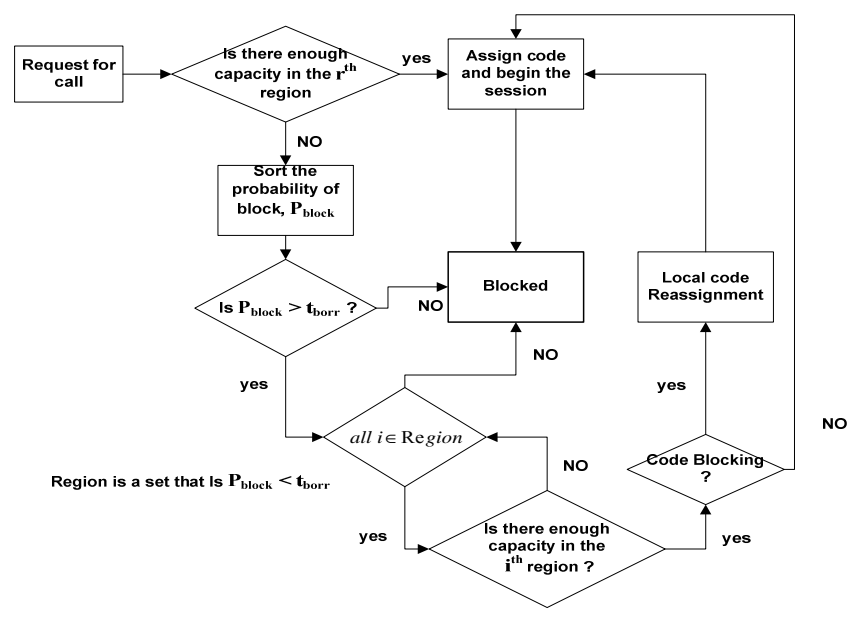

Fig. 6. HCA flow chart 


\section{G. Region Division Allocation (RDA)}

RDA [5] is a proactive code allocation scheme which divides the whole channelization code tree into regions before any call arrives. The code region is defined by the probability of requests for each data rate based on prior knowledge of the network's call distribution. Under RDA scheme, for all incoming calls, first the channel capacity is checked after which the call seeks for spreading code directly from its code region. If assignable code is available it is allocated and the call session begins if not then if the probability to borrow spreading code from other regions is less than its blocking probability then a code is borrowed from other regions otherwise the call is blocked

\section{OTHER CODE ALLOCATION SCHEMES}

As mentioned earlier several code allocation schemes were proposed to enhance efficient utilization of the channelization codes available. Some of these proposals are highlighted here.

\section{A. Efficient OVSF Allocation Considering the Dedicated Channel (DCH)}

In order to avoid code blocking code reassignment is mostly implemented. All the reassignment schemes or DCA consider that all the dedicated channels can be reallocated or reassigned as and when needed but in the real world this is not true because some DCH are delay sensitive. Therefore this unmovable $\mathrm{DCH}$ are as much as possible grouped together in the code tree [6]. A scheme called S-FCB is used. So when about to implement reassignment the sample of all the available codes including the movable codes are taken and the code that has minimum descendant code block is selected.

\section{B. An Interference Avoidance Code Assignment Scheme}

In this code allocation scheme apart from ascertaining the availability of channelization code but also the interference level of the system and the impact of the incoming call on the overall interference level is considered. Thus it is possible that a call is rejected not due to lack of assignable orthogonal code but because by allowing the session to begin the minimum interference level allowed will be exceeded [7]. It is very realistic that an incoming call adds to the already existing interference thus it is very important to consider the level and impact of MAI (Multiple Access Interference) to obtain a better performance

\section{Time Based Code Allocation Scheme}

In [8], the service time of most real time and multimedia applications such as video conferencing, video on demand and music downloading are known a priori, therefore the remaining time of each code occupied in the code tree is known. Thus if calls with similar remaining time are grouped together then a whole subtree of codes will be available at the same time thereby reducing to great extend code blocking. As a result more users can be accommodated.

\section{IMPROVED REGION DIVISION ALLOCATION (IRDA)}

In our algorithm as presented in Fig. 7, we introduce a notations call home code $C_{h}$ and visiting code $C_{v}$. By home code $C_{h}$, we mean a class of code reserved for certain group of call rates. A code becomes a visiting code $C_{v}$ when utilized by a call or session which initially is not meant for that code group. Therefore all visiting codes are engaged codes. As the algorithm shows, the first task of the base station is to check for available resources. Radio resource management (RRM) carry out the task of managing the effect of incoming calls on the overall interference level of the network and the quality of service requirement of the incoming call. If this requirements are met then the call checks for idle assignable spreading code in its $C_{h}$, if found it allocate it, if otherwise then other code classes are checked. If it ultimately finds an assignable code in any of the other code groups, code allocation takes place and session starts.

Meanwhile at the beginning of each frame all call sessions utilizing $C_{v}$ would look back at their home codes weather a code is free for use. If free, code reassignment takes place from visiting code to home code. If assignable (orthogonal) code is not found in all the regions, the call is blocked. In this work the reassignment is limited because for a particular session only a single code reassignment can take place which is the one taking place between it home and visiting code region.

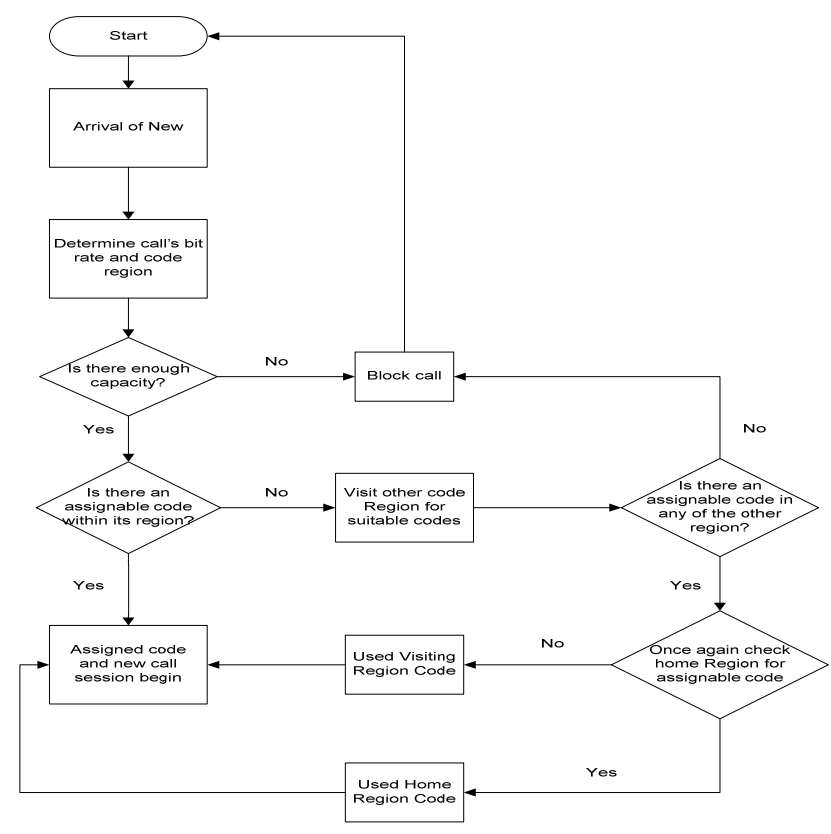

Fig. 7. IRDA flow chart

\section{Simulation AND RESUlt}

In our model, we considered four sets of call group ( $R, 2 R$, $4 R$ and $8 R$ ). Each of the rates is allotted equal number of OVSF code regions. The calls follow the poison's distribution. The incoming call is random and queue is first in first out (FIFO). As shown in the flow chart above, an incoming call is directed first to its code region to pick a code after verifying that there is enough capacity to accommodate 
the call. If the region have extra or remaining codes to allocate to the call, a new call session begins, if on the other hand there is no extra code, an assignable code is searched for in the other code regions. If available the call takes the code and the session begins. In a nutshell, our algorithm as clearly shown by our flow chart, allows a caller using a code in other regions not its allotted home region code to returns to its home immediately it find out that there is free code available. This idea also answers the need for fair allocation of codes between higher bit rate calls and lower bit rate calls. By changing the simulation time we can vary the amounts of calls. The simulation results for two different time duration is given, we observe that the higher the number of callers the higher the number of session accepted. We also observe that in either of the cases Improved RDA has lesser call drops compared to RDA.

Considering the results of the simulation contained in the Fig 8a and Fig 8b Improved RDA has a better performance with respect to RDA. The BLUE curve represents IMPROVED RDA and GREEN curve represents RDA. In both of the figures, RDA exhibit higher call drop for the same amount of traffic when compared to Improved RDA

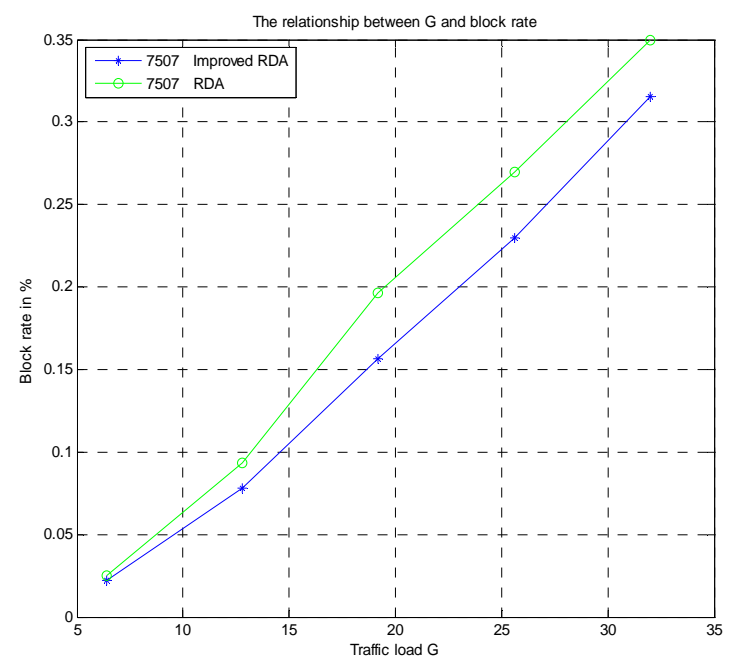

Fig. 8a. IRDA flow chart

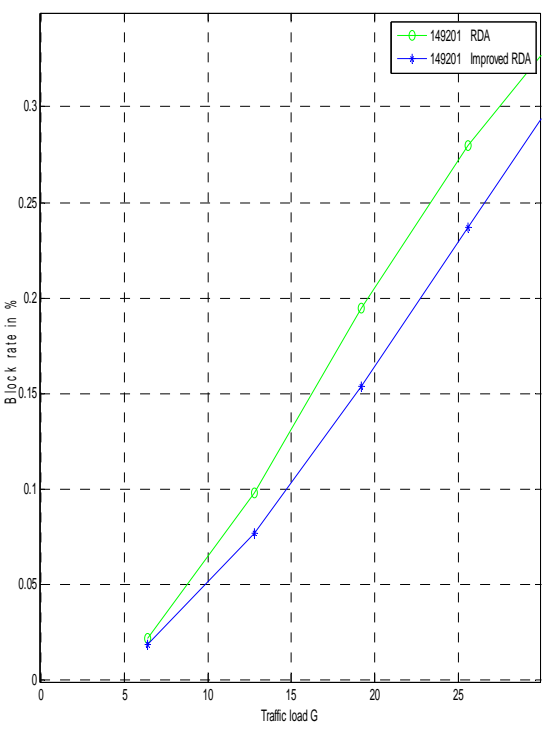

Fig. 8b. IRDA flow chart

\section{CONCLUSION}

Orthogonal Variable Spreading Factor (OVSF) code management and selection in the downlink of WCDMA is an important issue in the third generation CDMA network. In this research we proposed an improvement to Region Division Allocation scheme. Any in-coming call would first visit its pre-defined home region for codes within the code tree for its spreading code. The regions were predetermined depending on the data rate requirement of the in-coming call. The algorithm also allows a user to return to its home region if using codes from a visiting region at the beginning of each session of the call. With this improved code selection algorithm an improved usage of the available capacities and capabilities of the network are utilized. The simulation results as shown in the graphs indicate how our humble contribution helped in reducing code blocking, ensuring fair allocations amongst different bit rate callers and allowing more user elements UE to participate at the same time.

\section{REFERENCES}

[1] P. Lin, G. Chai-Hien and H. Ching-Chi, "OVSF code channel assignment with dynamic code set and buffering adjustment for UMTS," IEEE Transactions on Vehicular Technology, vol. 54, no. 2, pp. 591 - 602, Mar. 2005.

[2] T. Minn and K.-Y. Siu, "Dynamic assignment of orthogonal variable spreading factor codes in W-CDMA," IEEE Journal on Selected Areas in Communication, vo1.18, no. 8, pp.1429-1440, Aug. 2000.

[3] A. N. Rouskas and D. N. Skoutas, "Comparison of code reservation schemes at the forward link in WCDMA", in proc. 2002 4th International Workshop on Mobile and Wireless Communications Network, Sweden, 2002, pp. 191-195.

[4] Y. Sekine, K. Kawanishi, U. Yamamoto and Y. Onozato, "Hybrid OVSF code assignment scheme in W-CDMA," in proc. 2003 IEEE Pacific Rim Conferences on Communications, Computers and signal Processing, Canada, 2003, vol. 2, pp. 384-387.

[5] A. Rajipun, K. Kawanishi, U. Yamamoto, Y. Onozato and M. Matsushita, "Region division assignment of orthogonal variable spreading factor codes in W-CDMA," in proc. IEEE VTS $54^{\text {th }}$ Vehicular Technology Conference, USA, 2001, vol. 3, pp. 1884-1888.

[6] D. N. Skoutas and A. N. Rouskas, "Efficient selection of OVSF codes for the Downlink DCH in WCDMA," in proc. IEEE $59^{\text {th }}$ Vehicular Technology Conference, Italy, 2004, vol. 4, pp.1954-1958.

[7] L.-C. Wang, C.-W Chang and H. Huang, "An interference avoidance code assignment strategy for downlink multi-rate mc-ds-cdma tf-spreading," IEEE Transactions on Wireless Communications, vol. 6, no. 7, pp. 2508-2518, Jul. 2007.

[8] A. C. Kam, T. Minn, K.-Y. Siu, "Supporting rate guarantee and fair access for bursty data traffic in W-CDMA," IEE Journal on Selected Areas in Communications, vol. 19, no. 11, Nov. 2001.

[9] M. Karakoc, A. Soke and A. Kavak, "Using diploidy genetic algorithm for dynamic OVSF code allocation in WCDMA Networks," in proc. IEEE Radio and Wireless Symposium, USA, 2007, pp. 15-18.

[10] D. S Saini and S. V. Bhooshan, "Performance improvement in $3 \mathrm{G}$ and beyond CDMA systems using priority based code assignment scheme," in proc. International Conference on Signal Processing, Communications and Networking, India, 2007, pp. 98-101

[11] R. Assarut, K. Kawanishi, U. Yamamoto and Y. Onozato, "Performance evaluation of orthogonal variable-spreading-factor code assignment schemes based on UMTS/IMT-2000 in W-CDMA," in Proc. IEEE $56^{\text {th }}$ Vehicular Technology Conference Proceedings, Canada, 2002, vol. 3, pp. 1585-1589.

[12] 3rd Generation Partnership Project Technical Specification Group Radio Access Network, Spreading and modulation (FDD), 3GPP T.S. 25.213, V8.0.0-2008.

[13] R. L. Peterson, R. E. Ziemer and D. E. Borth, Introduction to Spread Spectrum Communication, 1st ed, New Jersey, USA: Prentice Hall, 1995, pp. $135-142$. 
[14] T. S. Rappaport, Wireless Communication: Principles and Practices, $2^{\text {nd }}$ Ed. New Jersey, USA: Prentice Hall PTR, 2002, pp. 34-39.

[15] A. Goldsmith, Wireless Communication, New York: Cambridge University press, 2005, pp. 403-450.

[16] M.R. Karim and M. Sarraf, W-CDMA and CDMA 2000 for 3G Mobile Networks, New York: McGraw-Hill, 2002, ch. 3, pp. 55-106.

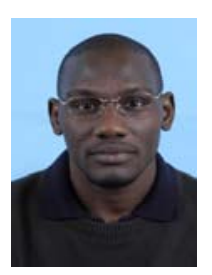

Mala Umar Mustapha Bakura was born in Bama, Nigeria in 1975. He received the B.Sc. Electrical and Electronic Engineering degree from the Islamic Institute of Technology (IIT) Dhaka Bangladesh now (Islamic University of Technology IUT) in 2000 and MSc in Communication Engineering from Beijing University of Post and Telecommunication (BUPT) in 2009, Beijing China. He is a staff of the department of Electrical and Electronics Engineering of University of Maiduguri, Nigeria from 2002 to date. $\mathrm{He}$ is presently a $\mathrm{PhD}$ student at BUPT. His research interests are wireless and Mobile communication

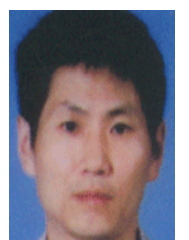

Xiaojun Jing, Professor, responsibility of the school of Information and Communication Engineering, Beijing University of Post and Telecommunication and tutor for doctoral candidates. Academic Pacemaker of Military Communication in BUPT, the chief of Biometrics Recognition and Multimedia Communication Laboratory.
He is honored with model demobilized and "Person of the Year 2008" of SCIENTIFIC CHINESE. He is a senior member of the Chinese Institute of Electronics, and judged as National High Technology Research and Development Program of China (863 Program). He received B.S. degrees from Beijing Normal University, M.S. and Ph.D. degrees from National University of Defense Technology, in 1995 and 1999, respectively. He pursued postdoctoral research between 2000 and 2002 in BUPT. He is the chairman of World Scientific and Engineering Academic and Society (WSEAS) in Athens, Greece, 2003 and in Florida, USA. 2004. His research interests are in the field of information security, image processing, and fingerprint recognition information fusion. He has organized and finished more than 20 national and provincial science research projects, such as 863 Program, the National Basic Research Program of China (973 Program), National Science Foundation of China (NSFC) and so on, with abundance of science research awards. He is currently working on 7 projects. Until now he has published more than 40 articles on famous magazines and important conference as the first author, more than 20 of which are accepted by SCI, EI search. Besides, He has published 8 books

Sun Songlin, An associate professor, IEEE Member. His main research interests includes: H.264 coding, multimedia communications such as CDN/IPTV, OFDM and signal processing, embedded systems such as DSP/FPGA/ARM. He has published more than 20 papers on famous magazines and conferences, and cooperated in 3 books with other authors. $\mathrm{He}$ has participated in more than 10 national and corporate projects. Current main research areas: wireless multimedia communications and signal processing, embedded system. 